\title{
Posttraumatische Belastungsstörung: Stand und Perspektiven des Wissens über effektive Therapien
}

\author{
A. Maercker \\ Klinische Psychologie und Psychotherapie, Technische Universität Dresden
}

Die Posttraumatische Belastungsstörung (PTB) ist immer noch eine vergleichsweise «junge» Diagnose. Trotz ihres «jugendlichen» Alters von 19 Jahren - nach Aufnahme in das damalige DSM-III von 1980 gerechnet - ist die Forschung zu diesem Thema äußerst produktiv, wenn man die internationale Literatur zur PTB betrachtet. In den führenden englischsprachigen psychiatrischen Fachzeitschriften übertrifft die Anzahl der Veröffentlichungen zur Epidemiologie, Pathogenese und Therapie der PTB derzeit die der anderen psychischen Störungsbilder. Dies sieht in der deutschsprachigen Fachliteratur und Forschungslandschaft (noch) anders aus, denn hier sind Publikationen zur PTB eher selten. Es ist deshalb zu begrüßen, daß die Zeitschrift «Verhaltenstherapie» ein Schwerpunktheft zur PTB ermöglicht hat.

In diesem Editorial versuche ich einen kurzen Überblick über den Stand des Wissens zu empirisch-validierten Therapieformen der PTB zu geben. Dieser Überblick stützt sich auf eine umfassende neuere Metaanalyse sowie einige brandneue kontrollierte Therapiestudien. Weiterhin werden einige Entwicklungs- und Innovationslinien skizziert, die sich aus der rasanten Tempo der PTB-Forschung für die Psychotherapie dieser Störung ergeben. Im Fall der PTB-Behandlung sind die Entwicklungen der psychotherapeutischen Methoden vielleicht von ganz besonderem Interesse, denn die Psychotherapien insgesamt zeigen sich in ihrer Wirkung den Pharmakotherapien überlegen. Dieses Ergebnis, über das aufgrund der bisherigen Befundlage [van Etten und Taylor, 1998; Otto et al., 1996; Sherman, 1998] ein Konsensus besteht, sollte ein starker Impuls sein, nach weiteren psychotherapeutischen Innovationen zum Wohle der Patienten zu suchen. Deutschsprachige bzw. europäische Beiträge hierfür werden noch gesucht, denn diese sind in der internationalen PTB-Forschung und -Therapie stark unterrepräsentiert.

Wie sehen die Therapieformen und die Ergebnisse der neuesten Metaanalyse [van Etten und Taylor, 1998] im einzelnen aus? Generell läßt sich feststellen, daß bisher eine ganze Reihe verschiedener Therapieformen für die PTB-Behandlung angewendet wurden. Die am häufigsten in kontrollierten Studien untersuchten Therapieformen gehören entweder direkt zur Verhaltenstherapie (z.B. In-sensu-Konfrontation, In-vivoKonfrontation), zur kognitiven Therapie bzw. kognitiv-behavioralen Kombinationen (z.B. Stress-Inokkulations-Training: SIT) oder wurden entscheidend aus ihnen heraus entwickelt (z.B. EMDR, dt.: Augenbewegungs- und DesensibilisierungsVerarbeitungstherapie).

Nach der behavioralen Konzeptualisierung verursacht das Erleben eines Traumas eine konditionierte Furcht- oder Angstreaktion in bezug auf traumarelevante Stimuli. Vermeidung und Rückzug von den traumarelevanten Stimuli wird in der Zeit nach der Traumaeinwirkung negativ verstärkt (d.h. durch das Ausbleiben negativer Konsequenzen verstärkt) und damit eine Habituationsreaktion verhindert. So bildet sich ein Symptombild heraus, das von weiteren Angstaktivationen (Intrusionen), von Vermeidung und von ängstlichem Hyperarousal gekennzeichnet ist. Die behaviorale Theorie im engeren Sinne kann aber nicht sehr gut die Spontaneität der Intrusionen sowie das sogenannte Numbing (allgemeiner emotionaler Taubheitszustand) erklären.

Die kognitiv-behavioralen Theorien der PTB betonen die Erwartungen und Bewertungen der persönlichen Bedeutung des Traumas. Bekannte Ansätze sind beispielsweise das Modell der emotionalen Verarbeitung nach Foa et al. [1989] sowie ähnliche Modelle [z.B. Chemtob et al., 1988; Ehlers und Steil, 1995]. In diesen Konzeptualisierungen werden veränderte kognitiv-emotional-physiologische Netzwerke bzw. Veränderungen der persönlichen Bedeutungszuschreibungen als Faktoren der Aufrechterhaltung der Störung beschrieben. Weitere Ansätze stellen bestimmte, typisch nach einem Trauma veränderte kognitive Schemata in den Mittelpunkt der Therapie, wie z.B. Sicherheits- und Intimitätsüberzeugungen [Resick und Schnicke, 1993].

Wie sieht nun die neueste verfügbare Metaanalyse der Behandlung chronischer PTB [van Etten und Taylor, 1998] konkret aus? Sie umfaßte 39 Therapiestudien mit insgesamt 61

\begin{tabular}{ll}
\hline KARGER & ( 1999 S. Karger GmbH, Freiburg \\
$\begin{array}{l}\text { Fax +49 761 45207 14 } \\
\begin{array}{l}\text { E-mail Information@Karger.de } \\
\text { www.karger.com }\end{array}\end{array}$ & $\begin{array}{l}\text { Accessible online at: } \\
\text { www.karger.com/journals/ver }\end{array}$
\end{tabular}

PD Dr. Dr. Andreas Maercker 
Behandlungsbedingungen. 19 Behandlungsbedingungen waren psychopharmakologisch, 13 verhaltenstherapeutisch-kognitiv, 11 EMDR, nur 3 weitere betrafen andere Psychotherapiemethoden (jeweils einmal Entspannung, Hypnose und psychodynamische Therapie). Die 13 verhaltenstherapeutischkognitiven Behandlungen setzten sich zusammen aus 10 Konfrontationstherapien (auch Exposition oder Flooding) sowie 2 Stress-Inokkulationstrainings- und einer Angst-ManagmentTherapie.

Im Ergebnis dieser Metaanalyse zeigte sich, daß die psychologischen Therapien signifikant geringere Abbrecherraten hatten als die Pharmakotherapien (14 vs. 32\%). Daneben zeigte sich, daß die psychologischen Therapien auch in der Symptomreduktion effektiver waren als die Pharmakotherapien. Innerhalb der Psychotherapieformen waren die Verhaltenstherapien und die EMDR-Methode den anderen Psychotherapieformen überlegen, unterschieden sich aber in ihrer Wirksamkeit nicht voneinander. Für die verhaltenstherapeutische Konfrontation und für EMDR ergaben sich Effektstärken zum Therapieende bei den PTB-Fragebogenwerten von etwa 1,25, d.h. einem durchschittlichen Patienten ging es nach Therapie besser als $90 \%$ der Patienten zu Therapiebeginn. In den 4-Monats-Katamnesen betrug diese Effektstärke für die Verhaltenstherapie 1,63 und für die EMDR-Methode noch 1,33. (Die Effektstärke von 1,63 entspricht hier, daß es einem Patienten nach der Therapie besser geht als 95\% der Patienten vor der Therapie).

Was ließ sich aus dieser Metaanalyse außer der zur Zeit feststellbaren Überlegenheit der Psychotherapie über die Pharmakotherapie ablesen? Zum einen, daß eine neue Methode, das EMDR, annähernd gleiche Effektivitätswerte wie die Verhaltenstherapie erreichte, wobei der 4-Monats-Katamnesezeitraum jedoch eine Überlegenheit der Verhaltenstherapie ergab. Für Schlußfolgerungen im weiteren Sinne ist das gute Ergebnis der EMDR-Methode durchaus interessant: Eine neue störungspezifische Therapiemethode entstand genuin im PTB-Bereich. Diese neue Methode ist ernst zu nehmen und in ihrer möglichen Innovationskraft für andere Psychotherapiefelder zu überprüfen. (Über einige Probleme der EMDRTheorie und die fragwürdige Marketingstrategie der Therapie siehe Maercker, 1998).

Zum anderen zeigte die Metaanalyse, daß die kognitiven PTB-Therapien im engeren Sinne, die auf eine In-sensu-Konfrontation mit dem Trauma verzichten, bisher nicht mit methodisch zufriedenstellenden Therapiestudien vertreten waren, wenn man vom Stress-Inokkulations-Trainings nach Meichenbaum und von eher unspezifischen AngstmanagmentTrainings absieht. Dieser unbefriedigende Stand hat sich allerdings in der letzten Zeit geändert.

Im folgenden soll über neuere Entwicklungs- und Innovationslinien auf dem Gebiet der PTB-Therapie berichtet werden, die hauptsächlich nach dem Abschluß der Datensammlung für die van Etten- und Taylor-Metaanalyse entstanden. Auf drei Entwicklungslinien wird dabei eingegangen:
- die Evaluation kognitiver Therapien;

- die Kombination von Verhaltenstherapie (In-sensu-Konfrontation) mit Familientherapie;

- einige neu entwickelte Therapieformen wie «Schreib-Therapien» und der «kognitiv-narrative Ansatz».

\section{Zur Evaluation kognitiver Therapien}

Marks et al. [1998] veröffentlichten eine kontrollierte Therapiestudie, in der sie ausschließlich kognitives Restrukturieren mit ausschließlicher Konfrontation (in sensu und in vivo), eine kombinierte kognitive und Konfrontationsbedingung sowie eine Kontrollbedingung verglichen. Das kognitive Restrukturieren wurde mit Beckschen Methoden durchgeführt, jede kognitive bzw. verhaltensmäßige Konfrontation mit den Traumaerlebnissen wurde ausgeschlossen. Im Ergebnis zeigten sich gleichermaßen positive Veränderungen für die kognitive, die Konfrontations- und die kombinierte Bedingung. Für die PTB-Symptomatik ergaben sich für alle drei Bedingungen Effektstärken von 1,0 bis 2,0 direkt nach der Therapie, die nach 6 Monaten Katamnese auf 1,2 bis 2,9 stiegen. Damit war eine Gleichrangigkeit des rein kognitiven Vorgehens mit der bisher etablierten In-sensu-Konfrontation belegt. Interessanterweise beschrieben die Autoren der Studie jedoch, daß die Durchführung der kognitiven Restrukturierung für die Therapeuten anstrengender war als die Konfrontationstherapie. Tarrier et al. [1999] verglichen ebenfalls eine rein kognitive Behandlungsbedingung (nach Beckschen Methoden sowie in Anlehnung an das Konzept der typischen poststraumatisch veränderten kognitiven Schemata nach Resick und Schnicke [1993]) mit einer In-sensu-Konfrontationstherapie. Hier fand sich ebenfalls kein statistischer Erfolgsunterschied zwischen beiden Therapieformen: Beide erreichten gute Therapieerfolge, die Effektstärken für einzelne Erfolgsmaße lagen allerdings niedriger bei $0,6-1,3$. Beide Studien zeigen zusammengenommen, daß auch rein kognitive Therapieformen, ohne die therapeutische Konfrontation mit dem Traumageschehen, effektiv sind.

\section{Zur Kombination von Therapieformen}

Als Beispiel für eine Therapiestudie, die die Konfrontationsbehandlung mit ganz anderen Therapieansätzen kombiniert, soll die Studie von Glynn et al. [1999] angeführt werden. Hier wurden Patienten mit chronifizierter PTB entweder einer alleinigen In-senu-Konfrontationstherapie, einer In-sensu-Konfrontationstherapie mit nachfolgenden Familientherapiesitzungen sowie einer Kontrollbedingung zugeordnet. Die Familientherapie war so konzipiert, daß mindestens ein weiteres Familienmitglied anwesend war und daß nach einem festgelegten Therapieplan (unter anderem Psychoedukation, Kommunikationstraining) interpersonelle Probleme der Patienten im Mittelpunkt standen. Zur Enttäuschung der Therapeuten und Autoren dieser Studie ergab sich, daß die gemessenen psychosozialen Anpassungswerte für beide Behandlungsbe- 
dingungen gleich waren und für beide nicht so gut wie aus der Behandlung von akuter PTB bekannt. Damit hatte sich gezeigt, daß sich also kein Vorteil der Kombination mit Familientherapie erreichen ließ (außer in einem Einzelwert für Problemlösefähigkeiten).

Die Kombinationsbehandlung von PTB und weiteren damit verbundenen psychosozialen Problemfeldern ist damit erst einmal als unbefriedigend anzusehen. Glynn et al. [1999] diskutierten, daß das Timing der Therapien nach einem Trauma bzw. eine andere Frequenz und Intensität der zusätzlichen Therapie zum enttäuschenden Ergebnis beigetragen haben könnte. Hier sind weitere Studien noch dringend erforderlich, denn in der therapeutischen Praxis wird ja oft von der Effektivität solcher und anderer Therapiekombinationen ausgegangen.

\section{Zur Entwicklung neuartiger Therapieformen}

Seit längerem gibt es die Annahme, daß sich das therapeutisch angeleitete Schreiben über das Trauma (writing assignment) als therapeutisch wirksam erweist. Einzelbefunde gab es dazu unter anderem von Greenberg und Stone [1992], Lange et al. [1997] und Pennebaker et al. [1990]. In einer Metaanalyse hat Smyth [1998] die Ergebnisse zu diesen «Schreibtherapien» zusammengefaßt, wobei allerdings auch nichtklinische Stichproben bzw. Stichproben mit Personen, die als Zielgröße z.B. schwere Arbeitsprobleme haben, zusammengefaßt wurden. Im Ergebnis zeigte sich, daß die Effektstärken für diese einzelne Therapieintervention im mittleren Bereich von 0,33-0,68 liegen. Dieses Ergebnis erreicht eine vergleichbare Höhe wie andere Psychotherapieerfolge bei anderen Störungen, auch wenn es geringer als die Ergebnisse der oben genannten kognitiv-behavioralen Therapien ist (die allerdings ja nicht nur eine einzelne Intervention umfassen). Es ist verständlich, daß aufgrund dieser vielversprechenden Ergebnisse einige Therapieforscher am zielgerichteten Einsatz dieser Intervention in der PTB-Therapie arbeiten [z.B. Lange, 1997].

Als letzte Innovationslinie in der PTB-Therapie soll der «konstruktiv-narrative» Ansatz von Meichenbaum [1994; 1999 in diesem Heft] genannt werden. Er setzt ebenfalls an der therapeutischen Wirkung des Berichtens über das Trauma an, an den spontanen Berichten der Patienten und ihren für diese Berichte gewählten Metaphern, Sinnbezüge und Bewältigungserfolge. Die Therapie zielt also nicht auf das posttraumatische Vermeidungsverhalten der Patienten (wie bei der Konfrontationsmethode und dem «Schreiben über das Trauma»), sondern im Sinne eines ressourcenorientierten Ansatzes auf die von den Patienten bisher gezeigten Bewältigungsintentionen und persönlichen Sinnsysteme. Die empirische Überprüfung des «konstruktiv-narrativen» Ansatzes steht allerdings noch aus.

Alle bisher berichteten Befunde bezogen sich auf Studien aus dem englischsprachigen Raum. Leider gibt es aus dem deutschsprachigen Raum noch keine publizierten kontrollier- ten Therapiestudien. Eine einzige kleinere Studie [Frommberger et al., 1998] verglich 10 Patienten mit einer verhaltenstherapeutischen Konfrontationsbehandlung mit 11 Patienten unter Medikamentengabe (Paroxetin). In beiden Gruppen verbesserte sich die PTB-Symptomatik signifikant.

Aufgrund der kaum vorhandenen therapiebezogenen deutschsprachigen Therapieuntersuchungen, widmet sich das vorliegende Heft einer Reihe weiterer wichtiger Aspekte der PTB-Therapien, die auch ohne das Vorliegen kontrollierter Therapiestudien relevant sein dürften. Poldrack und Kollegen [1999, in diesem Heft] haben die (neben sexuellen Gewalterlebnissen) hierzulande wahrscheinlich häufigste Traumatisierungsart zum Thema: die PTB bei Verkehrsunfallopfern. Nach 3 Monaten haben fast $40 \%$ der Patienten einer unfallchirurgischen Abteilung eine vollausgebildete PTB. Als wichtiger Prädiktor für die Entwicklung der PTB werden bestimmte Formen des (dysfunktionalen) Grübelns identifiziert; ein Ergebnis, daß für Frühinterventionen von Bedeutung sein könnte.

Donald Meichenbaum konnte dafür gewonnen werden, seinen neuen «konstruktiv-narrativen» Therapieansatz in einem Originalartikel zu erläutern [Meichenbaum, 1999, in diesem Heft]. Bei den oben berichteten guten Effektstärken für rein kognitive Therapien bzw. «Schreibtherapien» ist durchaus zu erwarten, daß dieser Therapieansatz seine Wirksamkeit erweist und eine sinnvolle Ergänzung des Therapiemethodenspektrums darstellt. In Dresden wird zur Zeit ein ähnlicher Therapieansatz («Life-Review-Therapie»), der eigentlich aus der Therapie von Altersdepressionen stammt, für PTB-Patienten adaptiert und in Behandlungen eingesetzt und untersucht [Maercker et al., 1999].

Zwei weitere Beiträge berichten über spezifische Problemfelder in der Therapie bei PTB, die möglicherweise wichtige intervenierende Variablen des Therapieerfolgs in der Praxis zum Thema haben: der Einsatz von Dolmetschern bei nichtdeutschsprachigen Patienten/Klienten (z.B. Flüchtlingen und Asylsuchenden in Deutschland) [Abdallah-Steinkopff, 1999, in diesem Heft] und die besondere Problematik von Männern aus Rettungs- und Hilfsberufen bei der Akzeptanz von Psychotherapieangeboten [Pieper und Maercker, 1999, in diesem Heft]. Abschließend werden im Beitrag von Boos et al. [1999, in diesem Heft] die empirischen Ergebnisse einer Therapieevaluation in einem stationären Gruppensetting einer Psychosomatischen Klinik beschrieben. Die Therapie beruht hierbei im wesentlichen auf dem kognitiven Ansatz von Resick und Schnicke [1993]. Die geschilderten Evaluationsergebnisse sind zufriedenstellend und zeigen vor allem eine Zeitstabilität über einen 3monatigen Katamnesezeitraum.

Die genannten Beiträge können vielleicht für Praktiker und Forscher eine Ermunterung sein, dem weiteren Wissens- und Kompetenzfortschritt des noch jungen PTB-Gebiets mit Spannung und Interesse entgegenzusehen. 


\section{Literatur}

Abdallah-Steinkopff B: Psychotherapie bei Posttraumatischer Belastungsstörung unter Mitwirkung von Dolmetschern. Verhaltenstherapie 1999;9:211-220. Boos A, Scheifling-Hirschbil I, Rüddel H: TherapieEvaluation einer stationären Gruppe «Traumabewältigung» innerhalb der psychosomatischen Behandlung und Rehabilitation von Patientinnen mit chronischer PTBS. Verhaltenstherapie 1999;9:200-210.

Chemtob C, Roitblat HL, Hamada RS, Carlson JG, Twentyman CT: A cognitive action theory of posttraumatic stress disorder. J Anxiety Dis 1988;2;253275.

Ehlers A, Steil R: Maintenance of intrusive memories in posttraumatic stress disorder: A cognitive approach. Behav Cognit Psychoth 1995;23;217-249.

Foa EB, Steketee G, Rothbaum BO: Behavioral/cognitive conceptualizations of post-traumatic stress disorder. Behav Ther, 1989;20;155-176

Frommberger U, Nyberg E, Richter H, Novelli-Fischer U, Stieglitz RD, Berger M: Comparison between paroxetine and behaviour therapy in patinets with posttraumatic stress disorder. Poster CINP conference, Glasgow 12.-16.07.1998.

Glynn SM, Eth S, Randolph ET, Foy DW et al: A test of behavioral familiy therapy to augment exposure for combat-related posttraumatic stress disorder. J Consult Clin Psy 1999;67;243-251.

Greenberg MA, Stone AA: Emotional disclosure about traumas and its relation to health: Effects of previous disclosure and trauma severity. J Pers Soc Psy 1992;63;321-329.
Lange A, Richard R, Kiestra J, van Oostendorp E: Cognitive treatment through positive self-verbalization: A multiple case study. Behav Cognit Psychother 1997;25;161-171.

Maercker A, Schneider A, Kahler J: Lebensrückblickstherapie: Eine kognitive Therapiemethode zur PTB-Behandlung Älterer. Dresden, Fachrichtung Psychologie der TUD 1999.

Maercker A: Nicht die Blicke heilen. EMDR - ein Wundermittel gegen Traumata? Psychol heute 1999;2;70-72.

Marks I, Lovell K, Noshirvani H, Livanou M, Thrasher S: Treatment of posttraumatic stress disorder by exposure and/or cognitive restructuring. A controlled study. Arch Gen Psychiatry 1998;55;317-325.

Meichenbaum D: A Clinical Handbook/Practical Therapist Manula for Assessing and Treating Adults with Posttraumatic Stress Disorder. Waterloo, Psychological Institute Press, 1994.

Meichenbaum D: Behandlung von Patienten mit posttraumatischen Belastungsstörungen: Ein konstruktivnarrativer Ansatz. Verhaltenstherapie 1999;9:186-189. Otto MW, Penana SJ, Pollack SJ, Smoller JW: Cognitive-behavioral and pharmacologic perspectivees on the treatment of post-traumatic stress disorder; in Pollack MH, Otto MW, Rosenbaum JF (eds): Challenges in Clinical Practice: Pharmacological and Psychosocial Strategies. New York, Guilford 1996.
Pennebaker JW, Colder M, Sharp LK: Accelerating the coping process. J Pers Soc Psy 1990;58;528-537.

Pieper G, Maercker A: Männlichkeit und Verleugnung von Hilfsbedürftigkeit nach berufsbedingten Traumata (Polizei, Feuerwehr, Rettungspersonal). Verhaltenstherapie 1999;9:222-229.

Poldrack A, Maercker A, Margraf J, Kloten D, Gavlik JM, Zwipp H: Posttraumatische Belastungssymptomatik und Gedankenkontrollstrategien bei Verkehrsunfallopfern. Verhaltenstherapie 1999;9:190-198.

Resick PA, Schnicke MK: Cognitive Processing Therapy for Rape Victims: A Treatment Manual. Newbury Park, Sage 1993.

Sherman JJ: Effects of psychotherapeutic treatments for PTSD: A meta-analysis of controlled clinical trials. J Traum Stress 1998;11;413-436.

Smyth JM: Written emotional expression: Effect sizes, outcome types, and moderating variables. J Consult Clin Psy 1998;66;174-184.

Tarrier N, Pilgrim H, Sommerfield C, Faragher et al: A randomized trial of cognitive therapy and imaginal exposure in the treatment of chronic posttraumatic stress disorder. J Consult Clin Psy 1999;67;13-18.

van Etten ML, Taylor S: Comparative efficacy of treatments for posttraumatic stress disorder: A meta-analysis. Clinic Psychol Psychotherapy 1998;5. 